\title{
View sensitivity increases for same-shape matches if mismatches show pairs of more similar shapes
}

\author{
REBECCA LAWSON \\ University of Liverpool, Liverpool, England
}

\begin{abstract}
Lawson, Bülthoff, and Dumbell (2003) found increased view sensitivity when more similar shapes had to be discriminated. Their sequential picture-picture matching studies showed depth-rotated views of novel, complex, 3-D objects. However, with a similar task and stimuli, Hayward and Williams (2000) found no variation in view sensitivity on same-shape match trials depending on whether similar or dissimilar pairs of shapes were shown on mismatch trials. Thus view sensitivity increases from dissimilarshape mismatches to similar-shape mismatches to same-shape matches (Lawson et al., 2003). However, view sensitivity may not increase on same-shape matches if those trials are combined with similarrather than dissimilar-shape mismatches (Hayward \& Williams, 2000). The latter result was reexamined here. Matches were view sensitive given even the dissimilar-shape mismatch context. This view sensitivity increased in the medium and especially the hard contexts that showed similar shapes on mismatches. Thus, indirectly increasing shape similarity via mismatch context modulated view-change effects (cf. Hayward \& Williams, 2000).
\end{abstract}

Our visual system must be able to recognize objects across a range of views. Theories of object recognition differ on whether they predict view sensitivity in many situations (e.g., Tarr \& Cheng, 2003) or view invariance under most circumstances (e.g., Biederman \& Gerhardstein, 1993). The evidence is mixed: Sometimes we are highly sensitive to the particular viewpoint from which an object is shown, but in other situations, performance is view invariant. It is therefore important to understand the conditions under which generalization across viewpoint is more or less efficient. One hypothesis proposed by theorists on both sides is that view sensitivity increases as the similarity of shapes that must be discriminated increases (e.g., Biederman \& Gerhardstein, 1993; Hamm \& McMullen, 1998; Tarr \& Cheng, 2003). Subordinatelevel recognition usually requires more similar shapes to be discriminated than does basic-level recognition (Lawson \& Jolicœur, 2003). Thus subordinate-level recognition of a particular animal as an Alsatian (not a labrador) is predicted to be more view sensitive than its basic-level recognition as a dog (not a cat). Biederman and Gerhardstein further proposed that though subordinate-level recognition may be view sensitive, basic-level recognition typically is not. However, Tarr and Cheng suggested that even everyday, entry-level recognition is view sensitive, but that view sensitivity increases when more similar shapes must be distinguished. Surprisingly, few studies have tested these claims.

This research was supported by a grant from the Leverhulme Trust. Correspondence should be sent to R. Lawson, Department of Psychology, University of Liverpool, Eleanor Rathbone Building, Bedford Street South, Liverpool L69 7ZA, England (e-mail: rlawson@liv.ac.uk).
Unfortunately, most studies to date have tested view sensitivity for a given type of stimuli at just one level of shape similarity. View sensitivity has usually been found when similar shapes must be distinguished (e.g., Bülthoff \& Edelman, 1992; Rock \& DiVita, 1987). In contrast, Biederman and Gerhardstein (1993) reported view invariance when shape discrimination was easier and, arguably, more like basic-level recognition, leading them to propose that everyday recognition is usually view insensitive. Note, though, that other studies presenting similar stimuli to Biederman and Gerhardstein have shown view sensitivity (e.g., Lawson \& Humphreys, 1996, 1998). The differences in view sensitivity across these studies might be due to differences in shape similarity (i.e., the difficulty of discriminating between shapes), but they might also be due to differences in the types of shapes presented (Hayward \& Williams, 2000; though see Johnson, 2001).

A direct test of whether increased shape similarity increases view sensitivity was reported recently by Lawson, Bülthoff, and Dumbell (2003). In four picture-picture matching studies, we found that sensitivity to depth rotation varied systematically with the shape similarity of the two objects depicted on a trial. On matches, both pictures depicted the same novel object. Here, performance was much worse if the two pictures showed different views of the object. Performance was less view sensitive on mismatches depicting two objects with similar shapes. Performance was view insensitive, with no difference between same- and different-view trials, when two dissimilar shapes were depicted.

Lawson et al. (2003) thus found that directly increasing shape similarity increased sensitivity to depth rotation for different trials presented to the same participant. 
All types of match and mismatch trials were mixed together in a block, so similarity was manipulated within participants. Similarly, both Lawson and Jolicœur (1998) and Murray (1998) found that directly increasing shape similarity increased sensitivity to plane misorientation in word-picture verification tasks.

However, there are two problems with such direct and within-participants manipulations of shape similarity. First, view sensitivity must be compared across trials that present different stimuli, since this is how shape similarity is varied directly. Effects on view sensitivity might then be due to differences in the stimuli presented. Second, view sensitivity may be overestimated if shape similarity is varied within participants (Hamm \& McMullen, 1998). An experiment may present only a few pairs of highly similar shapes that must be discriminated. Nevertheless this task demand may contaminate overall performance by encouraging participants to always use a slow, conservative, and view-sensitive identification process. This could result in view sensitivity on trials when shapes need not be identified so specifically.

To avoid these two problems, we used a context manipulation in which shape similarity was varied only indirectly and between participants. View sensitivity was compared across different participants shown the same shapes in identical match trials. Only mismatch context was varied across participants by mixing in either hard or easy mismatches that showed pairs of more or less similar shapes, respectively. This contrasts to direct manipulations of shape similarity, where view sensitivity is compared across trials that present different stimuli in such a way that shape discriminability differs across these trials themselves. If, instead, shape similarity increases only indirectly on mismatches, does view sensitivity on matches increase? Participants in the easy context saw dissimilar shapes only on mismatches. Given that they never had to discriminate between similar shapes, Biederman and Gerhardstein (1993) would not predict their performance to be view sensitive.

Recent context manipulation studies have varied shape similarity indirectly in tasks requiring the identification of plane-rotated views of familiar objects. Hamm and McMullen (1998) failed to find indirect similarity effects in a word-picture verification task. They varied wordpicture similarity on mismatch trials (e.g., for subordinatelevel verification, the word Alsatian followed by a picture of a labrador, parrot, or car for the hard, medium, and easy mismatches, respectively). For all groups, matches were view sensitive for subordinate-level verification (Alsatian-Alsatian) and view invariant for basiclevel verification ( $\log$-Alsatian), so directly varying shape similarity influenced view sensitivity. However, indirectly manipulating shape similarity had no effect in either case - shape similarity on mismatches did not affect view sensitivity on matches. In contrast, both Lawson (2003) and Nicholson and Humphrey (2001) found increased view sensitivity on matches when mismatches showed more similar shapes. This mismatch context effect was, though, transient with no carryover effects in
Lawson (2003) and occurred only for line drawings, not for colored, shaded images in Nicholson and Humphrey (2001). Thus increasing shape similarity indirectly on mismatches does not reliably influence sensitivity to plane rotation on matches.

Context manipulation studies have also presented depth-rotated views of novel objects in picture-picture matching tasks. View sensitivity was found unless stimuli could be identified using a view-invariant feature such as color (Hayward \& Williams, 2000) or the angle of a part (Vanrie, Beatse, Wagemans, Sunaert, \& Van Hecke, 2002; Vanrie, Willems, \& Wagemans, 2001). Importantly, Hayward and Williams reported that the degree of view sensitivity on matches was not influenced by the similarity context provided by mismatches. They concluded that view sensitivity is affected by mismatch similarity context only if view-invariant, distinguishing information is available; otherwise shape determines view sensitivity. However, Edelman (1995) reported a nonsignificant trend for increased view sensitivity when shape similarity increased.

Thus in particularly easy contexts, where view-invariant features are probably available to support recognition, view invariance has been found for plane misorientation (for basic-level verification with a small set of dissimilar items in Hamm \& McMullen, 1998) and for depth rotation (Hayward \& Williams, 2000; Vanrie et al., 2002; Vanrie et al., 2001). In harder contexts, performance is view sensitive. So far, though, only Lawson (2003) and Nicholson and Humphrey (2001) have found a significant effect of similarity context on the degree of view sensitivity. Furthermore, this effect of indirectly varying shape similarity was weak in Lawson (2003) and was found only for line drawings in Nicholson and Humphrey. Also, both studies tested plane misorientation, so these results may not generalize to depth rotations (Lawson, 1999; Lawson, Humphreys, \& Jolicœur, 2000). No interaction between the degree of view sensitivity on matches and mismatch similarity context was reported by Hamm and McMullen, Hayward and Williams, or Nicholson and Humphrey for shaded images. Thus directly varying shape similarity clearly affects view sensitivity (Lawson et al., 2003; Lawson \& Jolicœur, 1998; Murray, 1998). However, at best there is only weak evidence that the degree of view sensitivity varies depending on mismatch similarity context, as Tarr and Cheng (2003) predicted.

It may, though, be difficult to manipulate similarity context experimentally for familiar objects. People may try to discriminate such stimuli from all other objects that they know, even if only dissimilar shapes are shown. For example, people may not identify something as a dog until they are confident it is not a cat. This criterion may not readily be relaxed if an easier similarity context is provided (e.g., if a dog is the only animal presented in a study).

Similarity context may be easier to manipulate when novel objects are presented. Here, participants have fewer expectations about the similarity of stimuli in the study. Nevertheless, Hayward and Williams (2000) found no difference in the degree of view sensitivity for novel ob- 
jects across different contexts (though see Edelman, 1995). However, they tested all participants in all contexts, so carryover effects may have weakened any context effects. They also reported data averaged over test and context stimuli (personal communication, Hayward, February 2003). Variation in view sensitivity across these different types of stimuli may have masked differences in view sensitivity due to context.

The present context manipulation study presented novel objects in a picture-picture matching task and reexamined whether, in easier similarity contexts, view sensitivity to depth rotation is reduced, as Tarr and Cheng (2003) would predict, or eliminated, as Biederman and Gerhardstein (1993) would predict. The study used 20 sets of morphs, each derived from a single-object model such as a cannon or a roller skate. Each model was morphed to produce the start-point morph, S1. S1 was then morphed in another way to create morphs S2 to S13. All matches showed two pictures of S1 of a given object. These match trials were mixed with one of three sets of mismatch-context trials for three different groups of participants; see Figure 1. Hard mismatches showed two similar morphs (S1, S7) of the same object. Medium mismatches showed two less similar morphs (S1, S13) of the same object. Easy mismatches showed two S1 morphs of two different objects. Would view sensitivity on matches (identical across all three groups) be reduced for participants given the easy mismatches showing dissimilar shapes?

On match and mismatch trials, the two pictures could depict objects from the same or depth-rotated views. For generality, both small $\left(30^{\circ}\right)$ and large $\left(150^{\circ}\right)$ view changes were tested. Note, though, there is no simple relation between the size of this view change and the perceived effect on the stimulus. Certain view changes (such as those producing foreshortening) make identification much harder. Often, though, there is little effect of increasing the view change above around $30^{\circ}-45^{\circ}$ (Foster \& Gilson, 2002; Lawson \& Humphreys, 1996). I therefore did not predict a difference between the two view changes tested here.

\section{METHOD}

\section{Participants}

Thirty-six undergraduate students from the University of Liverpool took part in the study for course credit.

\section{Materials}

Textured, shaded, 3-D models of familiar objects were individually morphed in varied, arbitrary ways to generate a set of $20 \mathrm{~S} 1$ morphs (see Lawson et al., 2003, for details). S1 was then morphed to produce a series of shapes, S2 to S13, where the shape of S13 was least similar to that of S1. For each morph, grayscale pictures were produced of $30^{\circ}, 60^{\circ}$, and $240^{\circ}$ views (relative to the experimenterassigned $0^{\circ}$ front view) by horizontally rotating the morph in depth about the vertical axis running through its midpoint. Stimuli were presented against a black background inside a window measuring $450 \times 450 \mathrm{~mm}$ on the computer screen and subtended a visual angle of approximately $5^{\circ}$.

\section{Design}

All participants completed one block of 120 trials. Stimuli from each of the 20 morph sets were depicted in the first picture on three match and three mismatch trials. Each of these three trials presented a different-view condition: Relative to the first picture, the second picture was depth rotated by $0^{\circ}, 30^{\circ}$, or $150^{\circ}$. In the easy-context mismatches, a different object was presented in each of these three view conditions. On all trials, the first picture shown was the $30^{\circ}$ view of S1 of a given object. On matches, the second picture depicted S1 of that object. On mismatches, it showed S7 of that object, S13 of that object, or S1 of a different object in the hard, medium, and easy contexts, respectively. Twelve participants were assigned to each context; only the mismatches differed across these three groups.

\section{Apparatus and Procedure}

The experiment was run on a Macintosh PowerPC G4 computer using PsyScope Version 1.2.5 software. Participants decided whether two successive pictures showed the same or different objects and responded with a speeded "m" or "z" keypress, respectively. On each trial, "Get ready for the next trial" appeared for $750 \mathrm{msec}$ then, after $500 \mathrm{msec}$, the first picture was presented 50 pixels above and to the right of fixation for $500 \mathrm{msec}$. After a blank interval of $400 \mathrm{msec}$, the second picture was presented at fixation until the participant responded. The correct response was then given as feedback for $500 \mathrm{msec}$. There was an intertrial interval of $750 \mathrm{msec}$. Participants were told to ignore any difference in the view depicted in the first and second pictures. Trials were presented in a different, random order for each participant. Participants started with 20 practice trials selected at random from the experimental trials.

\section{RESULTS}

Response latencies less than $300 \mathrm{msec}$ or exceeding $2,300 \mathrm{msec}$ were discarded as errors (less than $1 \%$ of trials). No participants were replaced. There were nine empty cells in the by-items analyses, which were replaced by the mean for that condition. Results for byparticipants and by-items analyses are reported using subscripts ( $F_{\mathrm{p}}$ and $F_{\mathrm{i}}$ respectively). Analyses of variance (ANOVAs) were conducted on the mean reaction times (RTs) on correct match and mismatch trials and on the percentage of errors (Figures 2 and 3 ). There was one within-participants factor, view change $\left(0^{\circ}, 30^{\circ}\right.$, and $150^{\circ}$ ), and one between-participants factor, mismatch context (hard, S1-S7; medium, S1-S13; and easy, different shapes). Unless noted, all differences described below were significant $(p<.05)$ in by-participants and by-items post hoc Newman-Keuls analyses.

\section{Match Trials}

View change was significant for RTs $\left[F_{\mathrm{p}}(2,66)=\right.$ $\left.88.42, p<.001 ; F_{\mathrm{i}}(2,38)=150.12, p<.001\right]$ and errors $\left[F_{\mathrm{p}}(2,66)=75.98, p<.001 ; F_{\mathrm{i}}(2,38)=53.85, p<\right.$ $.001]$. Same-view matches (603 msec, $2.8 \%$ errors) were easier than $30^{\circ}$ and $150^{\circ}$ view-change matches $(836 \mathrm{msec}$, $21.8 \% ; 871 \mathrm{msec}, 25.7 \%$, respectively).

Mismatch context was significant for RTs $\left[F_{\mathrm{p}}(2,33)=\right.$ $\left.7.87, p<.002 ; F_{\mathrm{i}}(2,38)=153.62, p<.001\right]$ and errors $\left[F_{\mathrm{p}}(2,33)=65.06, p<.001 ; F_{\mathrm{i}}(2,38)=52.93, p<\right.$ $.001]$. The easy context $(618 \mathrm{msec}, 3.3 \%)$ was easier than the medium context (782 msec, 16.7\%), which in 


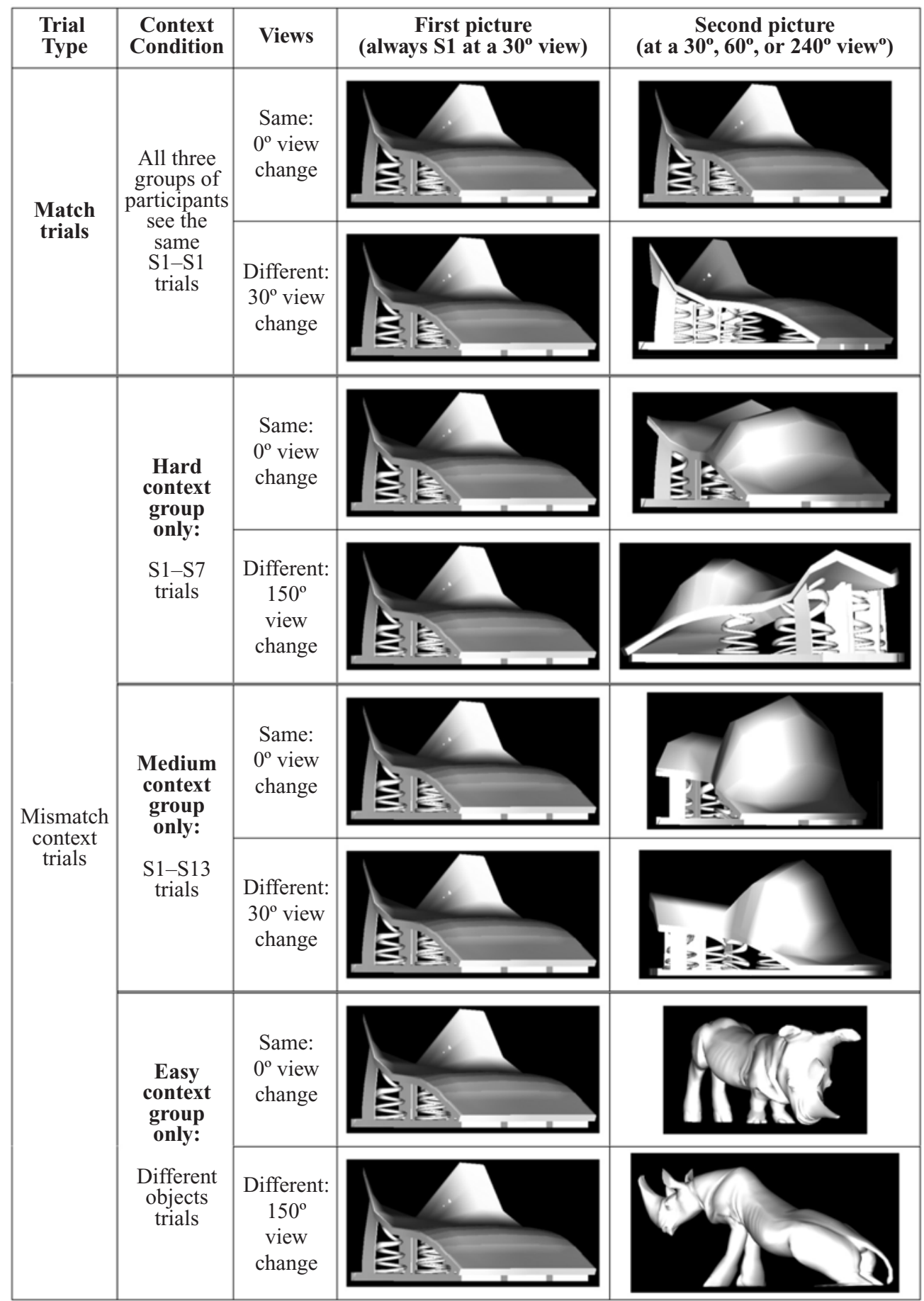

Figure 1. Examples of stimuli from the wedge-spring morph set for the eight types of trials: same-view and different-view conditions for match trials and for hard, medium, and easy mismatch-context trials. For different-view trials, examples of both $30^{\circ}$ and $150^{\circ}$ view changes are shown. Participants all saw identical same-shape match trials but saw just one type of different-shape mismatch-context trial (easy, medium, or hard). Shape similarity on matches was manipulated only indirectly, between participants, by showing different mismatches to different groups. View sensitivity on the identical match trials was then compared across the three groups of participants given hard, medium, or easy mismatch contexts. In contrast, Lawson, Bülthoff, \& Dumbell (2003) manipulated shape similarity directly, within participants, by presenting same-shape matches and different-shape (easy, medium, and hard) mismatches mixed together in a block. Lawson et al. (2003) then compared view sensitivity across the different types of match and mismatch trials. The stimuli tested here comprised the 18 stimuli presented in Experiments 3 and 4 of Lawson et al. (2003) plus Objects 5 and 12 from that paper; sample views of all the stimuli are shown in Figure 1 of that paper. 


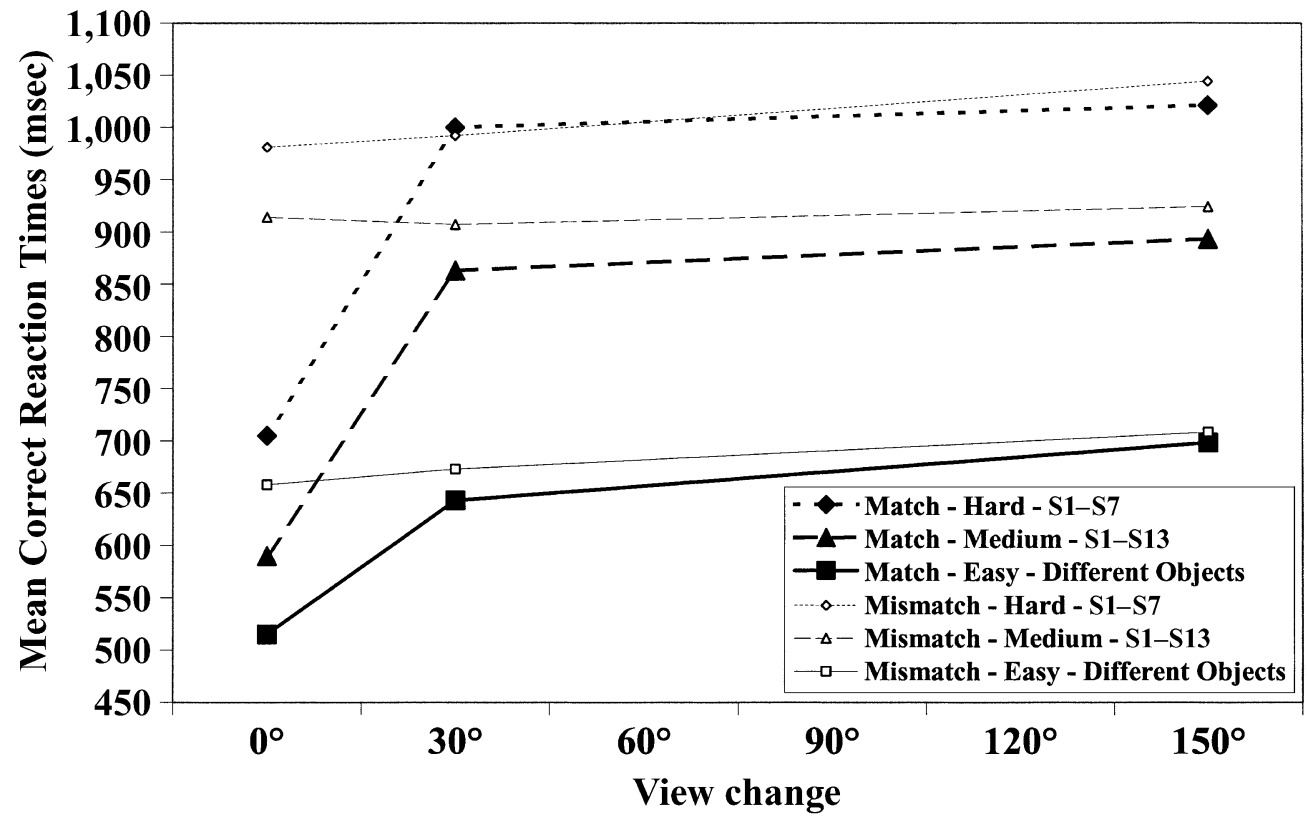

Figure 2. Mean reaction times on correct match trials and correct mismatch-context trials in the hard (S1-S7), medium (S1-S13), and easy (different object) contexts for $0^{\circ}, 30^{\circ}$, and $150^{\circ}$ view-change trials.

turn was easier than the hard context (909 msec, 30.3\%; not significant for RTs for participants).

Most important, the view-change $\times$ mismatch-context interaction was significant for both RTs $\left[F_{\mathrm{p}}(4,66)=3.22\right.$, $\left.p<.02 ; F_{\mathrm{i}}(4,76)=7.35, p<.001\right]$ and errors $\left[F_{\mathrm{p}}(4,66)=\right.$ $\left.23.69, p<.001 ; F_{\mathrm{i}}(4,76)=29.30, p<.001\right]$. Comparing view-change effects, in the easy context, same-view matches ( $515 \mathrm{msec}, 3 \%$ errors) were faster but no more accurate than $30^{\circ}$ and $150^{\circ}$ view-change matches $(643 \mathrm{msec}$, $2 \% ; 698 \mathrm{msec}, 5 \%$, respectively). In the medium context, same-view matches (590 msec, $2 \%)$ were both faster and more accurate than $30^{\circ}$ and $150^{\circ}$ view-change matches

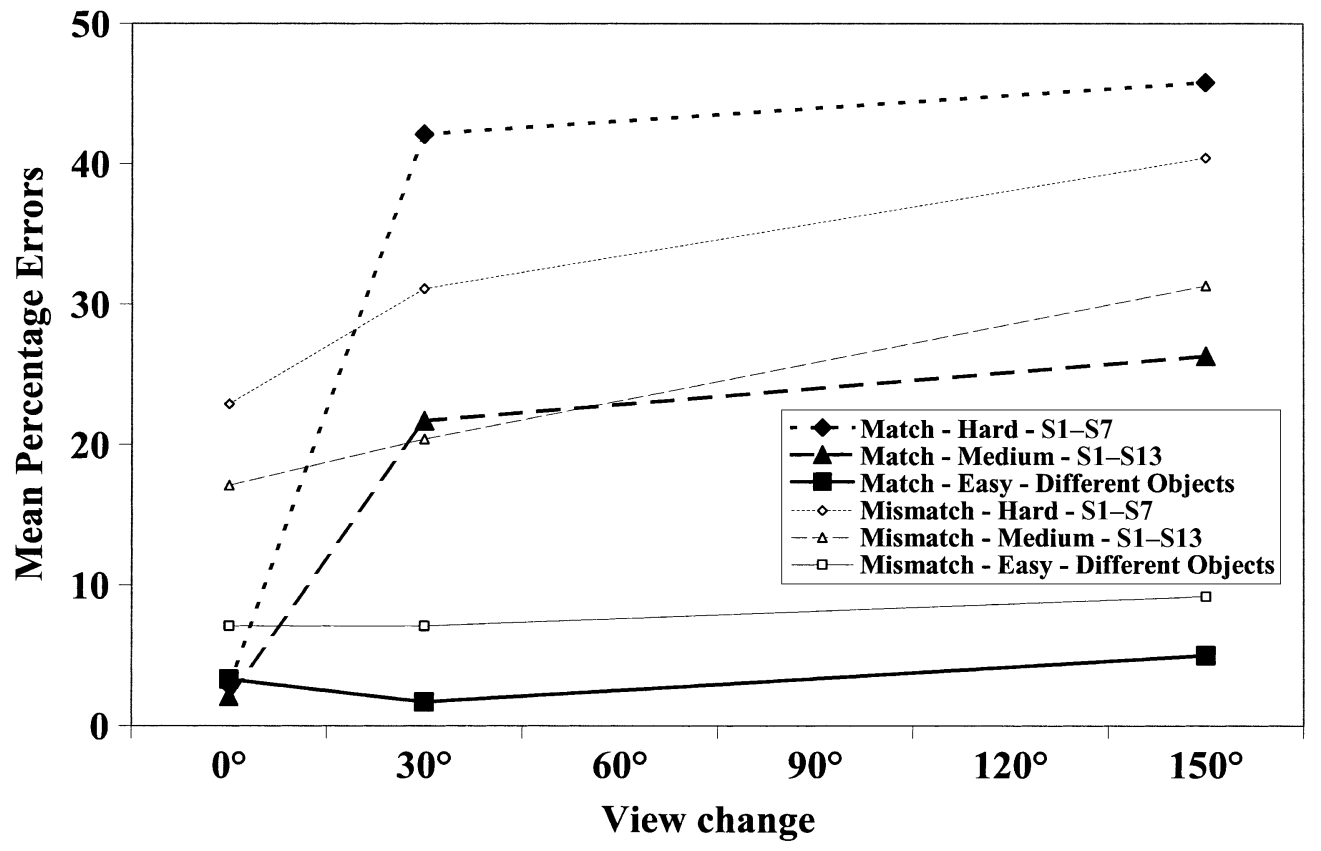

Figure 3. Mean percentage errors on match trials and mismatch-context trials in the hard (S1-S7), medium (S1-S13), and easy (different object) contexts for $0^{\circ}, 30^{\circ}$, and $150^{\circ}$ view-change trials. 
(863 msec, 22\%; $893 \mathrm{msec}, 26 \%$, respectively). Likewise, in the hard context, same-view matches $(705 \mathrm{msec}$, $3 \%$ ) were both faster and more accurate than $30^{\circ}$ and $150^{\circ}$ view-change matches $(1,000 \mathrm{msec}, 42 \% ; 1,021 \mathrm{msec}$, $46 \%$, respectively).

Thus view-change effects in the easy context occurred only for RTs (not for errors), with a difference of 156 msec and $0 \%$ errors between same- and different-view matches. These view-change effects increased greatly for both RTs and errors in the medium context ( 288 msec, $22 \%$ ) and increased further for errors in the hard context (306 msec, 41\%). This interaction could be interpreted as being due to a floor effect artifactually reducing view sensitivity in the easy-context condition. This, though, is inconsistent with Lawson et al. (2003) and Lawson and Bülthoff's (2004) findings of a similar interaction. Here, again, view sensitivity increased in the high-similarity conditions, but now these conditions were faster than the low-similarity conditions, so the results could not be due to floor effects. Note further that the view-change $\times$ mismatch-context interaction for errors was still significant when only the hard and medium contexts were compared $\left[F_{\mathrm{p}}(2,44)=7.27, p<.002\right.$; $\left.F_{\mathrm{i}}(2,38)=10.76, p<.001\right]$.

Comparing context effects, same-view matches in the easy context were faster (but not more accurate) than in the medium context, where, in turn, they were faster (but not more accurate) than in the hard context. In contrast, $30^{\circ}$ and $150^{\circ}$ different-view matches in the easy context were both faster and more accurate than in the medium context, where, in turn, they were both faster and more accurate than in the hard context. Thus, context effects on same-view matches occurred only for RTs (not for errors), with a difference of $190 \mathrm{msec}$ and $0 \%$ errors between the easy and hard contexts. Context effects on differentview matches increased greatly for both RTs and errors (340 msec, 41\%).

\section{Mismatch-Context Trials}

View change was significant for RTs $\left[F_{\mathrm{p}}(2,66)=3.94\right.$, $\left.p<.03 ; F_{\mathrm{i}}(2,38)=3.63, p<.04\right]$ and errors $\left[F_{\mathrm{p}}(2,66)=\right.$ $\left.14.66, p<.001 ; F_{\mathrm{i}}(2,38)=8.94, p<.001\right]$. Both sameview ( $851 \mathrm{msec}, 15.7 \%$ errors) and $30^{\circ}$ view change (857 msec, 19.9\%) mismatches were faster and more accurate than $150^{\circ}$ view-change mismatches $(892 \mathrm{msec}$, $26.9 \%)$.

Mismatch context was significant for RTs $\left[F_{\mathrm{p}}(2,33)=\right.$ $\left.8.39, p<.002 ; F_{\mathrm{i}}(2,38)=141.81, p<.001\right]$ and errors $\left[F_{\mathrm{p}}(2,33)=29.89, p<.001 ; F_{\mathrm{i}}(2,38)=22.47, p<\right.$ $.001]$. The easy context (680 msec, $7.8 \%)$ was easier than the medium context (915 msec, $22.9 \%$ ), which in turn was easier than the hard context $(1,005 \mathrm{msec}, 31.8 \%$; for RTs, not significant for participants).

The view-change $\times$ mismatch-context interaction was not significant for RTs $\left[F_{\mathrm{p}}(4,66)=0.52, p>.7 ; F_{\mathrm{i}}(4,76)=\right.$ $0.42, p>.7]$ and was significant over participants only for errors $\left[F_{\mathrm{p}}(4,66)=2.71, p<.04 ; F_{\mathrm{i}}(4,76)=1.82, p>.1\right]$. This is not considered further; it was not the focus of this study since, unlike the case for matches, different stimuli were presented to different participants on mismatches.

\section{DISCUSSION}

First, even in the easy similarity context, when mismatches showed two completely different shapes, performance was clearly better for same-view than for viewchange matches. This suggests that sensitivity to depth rotation will be pervasive in everyday, basic-level recognition since nearly all familiar objects have neighbors at least as similar in shape as in the easy mismatches here (see Biederman \& Gerhardstein, 1993).

Second, this view sensitivity to depth rotation on matches greatly increased as mismatch shape similarity increased in the medium context and especially in the hard context. There was not simply a dichotomy between view-invariant and view-sensitive performance on match trials across the different similarity contexts (cf. Hayward \& Williams, 2000; Vanrie et al., 2002; Vanrie et al., 2001). Instead, the results support Tarr and Cheng's (2003) prediction that the degree of view sensitivity increases as shape similarity increases. These results complement those of Lawson et al. (2003) by showing that indirectly as well as directly manipulating shape similarity increases view sensitivity to depth rotation. This suggests that view sensitivity will increase for the identification of objects with similarly shaped neighbors (e.g., dog, goat, deer) and at the subordinate level when finer shape discrimination is usually necessary (Lawson, 2003).

As in Hayward and Williams's (2000) studies, shaded, depth-rotated views of novel, complex, 3-D objects were presented in a sequential picture-picture matching task. One possible explanation for the present failure to replicate their null result is that the context- and view-change manipulations were at least twice as strong in the present study. Also, as discussed in the introduction, carryover context effects and averaging artifacts may have reduced Hayward and Williams's ability to detect effects of mismatch similarity context on the view sensitivity of match trials.

The present results suggest that view sensitivity is not determined primarily by the shape of objects, as Hayward and Williams (2000) proposed. In some situations, stimulus shape and familiarity probably have important effects on the view sensitivity of object identification, but these factors could not account for the present results. Here, shape similarity on mismatch context trials influenced view sensitivity on match trials even though these match trials were identical across the different contexts and so depicted identical shapes. Given a lifetime of recognizing familiar objects such as dogs and chairs, people may not readily relax their identification criterion if they are given an easier shape similarity context than that required in everyday recognition (Lawson, 2003). This problem was avoided in this study through testing novel objects.

The present results showed, first, view sensitivity to depth rotation even when only dissimilar shapes had to 
be discriminated, in a similarity context at least as easy as that found in basic-level object recognition. This finding fails to support Biederman and Gerhardstein's (1993) claim that everyday object recognition is typically view invariant. Second, increasing the shape similarity of objects shown on mismatch-context trials increased view sensitivity on match trials, failing to replicate Hayward and Williams's (2000) finding that view sensitivity was not influenced by similarity context. Instead, this pattern of results supports Tarr and Cheng's (2003) proposal that view sensitivity is found in most situations, with view sensitivity increasing when more similar shapes need to be discriminated.

\section{REFERENCES}

Biederman, I., \& Gerhardstein, P. C. (1993). Recognizing depthrotated objects: Evidence for 3-D viewpoint invariance. Journal of Experimental Psychology: Human Perception \& Performance, 19, 1162-1182.

Bülthoff, H. H., \& Edelman, S. (1992). Psychophysical support for a 2-D view interpolation theory of object recognition. Proceedings of the National Academy of Science, 89, 60-64.

EDELMAN, S. (1995). Class similarity and viewpoint invariance in the recognition of 3D objects. Biological Cybernetics, 72, 207-220.

Foster, D. H., \& Gilson, S. J. (2002). Recognizing novel threedimensional objects by summing signals from parts and views. Proceedings of the Royal Society of London: Series B, 269, 1939-1947.

Hamm, J. P., \& McMullen, P. A. (1998). Effects of orientation on the identification of rotated objects depend on the level of identity. Journal of Experimental Psychology: Human Perception \& Performance, 24, 413-426.

HAYWARD, W. G., \& Williams, P. (2000). Viewpoint dependence and object discriminability. Psychological Science, 11, 7-12.

JoHnson, S. H. (2001). Seeing two sides at once: Effects of viewpoint and object structure on recognizing three-dimensional objects. Journal of Experimental Psychology: Human Perception \& Performance, 27, 1468-1484.

LAWSON, R. (1999). Achieving visual object constancy over plane rotation and depth rotation. Acta Psychologica, 102, 221-245.
LAWSON, R. (2003). The effects of context on learning to identify planemisoriented views of familiar objects. Visual Cognition, 10, 795-821.

Lawson, R., \& BülthofF, H. H. (2004). Comparing view sensitivity in shape discrimination to shape sensitivity in view discrimination. Manuscript submitted for publication.

Lawson, R., Bülthoff, H. H., \& Dumbell, S. (2003). Interactions between view changes and shape changes in picture-picture matching. Perception, 32, 1465-1498.

LAWSON, R., \& HuMPHREYS, G. W. (1996). View-specificity in object processing: Evidence from picture matching. Journal of Experimental Psychology: Human Perception \& Performance, 22, 395-416.

LAWSON, R., \& HumphreYs, G. W. (1998). View-specific effects of depth rotation and foreshortening on the initial recognition and priming of familiar objects. Perception \& Psychophysics, 60, 1052-1066.

LaWson, R., Humphreys, G. W., \& Jolicceur, P. (2000). The combined effects of plane disorientation and foreshortening on picture namingOne manipulation or two? Journal of Experimental Psychology: Human Perception \& Performance, 26, 568-581.

LAWSON, R., \& Jolicceur, P. (1998). The effects of plane rotation effects on the recognition of brief masked pictures of familiar objects. Memory \& Cognition, 26, 791-803.

LAWSON, R., \& JOLICEUR, P. (2003). Recognition thresholds for planerotated pictures of familiar objects. Acta Psychologica, 112, 17-41.

MurRaY, J. E. (1998). Is entry-level recognition viewpoint invariant or viewpoint dependent? Psychonomic Bulletin \& Review, 5, 300-304.

Nicholson, K. G., \& HumphreY, G. K. (2001). Surface cues reduce the latency to name rotated images of objects. Perception, 30, 10571081.

Rock, I., \& DiVita, J. (1987). A case of viewer-centered object perception. Cognitive Psychology, 19, 280-293.

TARR, M. J., \& CHENG, Y. D. (2003). Learning to see faces and objects. Trends in Cognitive Sciences, 7, 23-30.

Vanrie, J., Beatse, E., Wagemans, J., Sunaert, S., \& Van Hecke, P. (2002). Mental rotation versus invariant features in object perception from different viewpoints: An fMRI study. Neuropsychologia, $\underline{\mathbf{4 0}}$, 917-930.

VanRIE, J., Willems, B., \& Wagemans, J. (2001). Multiple routes to object matching from different viewpoints: Mental rotation versus invariant features. Perception, 30, 1047-1056.

(Manuscript received April 23, 2003; revision accepted for publication December 1, 2003.) 